\title{
ON PSEUDO-DISTRIBUTIVE NEAR-RINGS
}

\author{
by GORDON MASON
}

(Received 17th February 1984)

\section{Introduction}

If $G$ is a group and $N$ a ring, the elements of the group ring $N G$ can be thought of either as formal sums $\sum n_{g} g$ or as functions $\phi: G \rightarrow N$ with finite support. If $N$ is a nearring, problems arise in trying to construct a group near-ring either way. In the first case, Meldrum [7] was able to exploit properties of distributively generated near-rings $(N, S)$ to build free $(N, S)$-products and hence a near-ring analogue of a group ring. For the latter case, Heatherly and Ligh [3] observed that the set of functions could be made into a near-ring under multiplication given by $\left(\phi^{*} \alpha\right)(g)=\sum_{x \in G} \phi(x) \alpha\left(x^{-1} g\right)$, provided $N$ satisfies

$$
\text { (P1) } a_{1} b_{1}+a_{2} b_{2}=a_{2} b_{2}+a_{1} b_{1}
$$

and

$$
\text { (P2) } n \sum_{1}^{k} a_{i} b_{i} \quad=\sum_{1}^{k} n a_{i} b_{i}
$$

for all $a_{i}, b_{i}, n \in N$ and $k \in Z^{+}$. Such near-rings are called pseudo-distributive. In fact these are precisely the conditions under which the set $N_{k}$ of $k \times k$ matrices over $N$ is also a near-ring and then both $N G$ and $N_{k}$ are pseudo-distributive.

Examples are found in [3], where some restrictions are given for a near-ring to be pseudo-distributive and yet not a ring (a so-called non-ring). In particular, $N$ should not contain an identity. On the other hand, any non-unital ring is a pseudo-distributive near-ring so the results of this paper will apply to non-unital rings (group rings and matrix rings). Moreover, we shall see that our near-rings have close connections to appropriate rings.

In the next section we develop some general facts about pseudo-distributive nearrings. In the third section we examine the structure of matrix near-rings and group nearrings. The basic reference for near-rings is [9], and in this paper all near-rings will be zero-symmetric right near-rings. For future reference we repeat the definition of an ideal: A subgroup $(I,+)$ of $(N,+)$ is an ideal of $N$ if it satisfies

(I1) $I$ is a normal subgroup

(I2) $n(x+a)-n x \in I$ for all $n, x \in N, a \in I$

(I3) $I N \subseteq I$.

We note that because of the zero symmetry $N I \subseteq I$ also, by (I2). 


\section{Pseudo-distributive near-rings}

As might be expected, a near-ring which is "almost abelian" (P1) and "almost distributive" (P2) has lots of rings associated with it. The proof of the following is straight-forward:

Proposition 2.1. If $N$ is pseudo-distributive, the following are rings:

(a) $N a=\{n a \mid n \in N\}$ for all $a \in N$.

(b) $N^{2}=\left\{\sum_{1}^{k} a_{i} b_{i} \mid a_{i}, b_{i} \in N\right\}$.

(c) $B(N)$, the set of central idempotents (with addition given by $e \oplus f=e+f-2 e f$ ).

(d) $N / I$ for any modular ideal $I$.

(e) $N / A$ where $A=\operatorname{Ann}^{l} N=\{n \mid n N=0\}$.

Note that $A$ is a non-zero ideal ([3]) and $N a$ and $N^{2}$ are also $N$-subgroups of $N$. Also $N^{2}$ satisfies (I2) and (I3) so would be an ideal iff it were a normal subgroup of $N$. In fact, this is true of any subgroup $J$ with $N^{2} \subseteq J \subseteq N$, i.e. any such normal subgroup $J$ is an ideal.

Following [5] let $[N, N]$ be the $N$-commutator ideal, namely the ideal generated by $S=\{n(a+b)-n a-n b\}$. Now in [5, Proposition 2.2] it was shown that the subgroup generated by $S$ satisfies (I2), and clearly (I3) holds (in fact $S N=0$ ) so again this subgroup lacks only normality to be an ideal. Maxson [6] has defined $D(N)$, the distributor ideal, to be the ideal generated by $T=\{n(a+b)-n b-n a\}$. By $\mathrm{P} 2, S=T$ in our case so $D(N)=[N, N]$. Letting $N^{\prime}$ be the commutator subgroup of $(N,+)$, we have from [2] that when $N$ is distributive, $N^{\prime}$ is an ideal and $N^{\prime} \cdot N=N \cdot N^{\prime}=0$. For $N$ pseudo-distributive, $N^{\prime} \cdot N=0$ still holds.

Proposition 2.2. If $N$ is pseudo-distributive, $C(N)=N^{\prime}+D(N)$ is an ideal and $N / I$ is a ring iff $I \supseteq C(N)$.

Proof. Since $N / D(N)$ is distributive, its commutator subgroup is an ideal which has the form $C(N) / D(N)$ for some ideal $C(N)$ of $N$. In fact $(N / D(N))^{\prime}=N^{\prime}+D(N) / D(N)$. Then $N / C(N)$ is isomorphic to the distributive near-ring $N / D(N)$ modulo its commutator and so is a ring. If $I$ is an ideal of $N$ for which $N / I$ is a ring, then the abelian addition and distributivity of $N / I$ shows that $N^{\prime} \subseteq I$ and $D(N) \subseteq I$ respectively, and conversely.

We shall call any ideal $I \supseteq C$ a ring ideal of $N$. For example, if $N$ is abelian then $C=D(N)$, and if $N$ is distributive then $C=N^{\prime}$. In fact in [2], Heatherly shows how nilpotent groups of class 2 can be made into distributive near-rings by defining $a \cdot b=$ $a+b-a-b$. Then $C=N^{\prime}=N^{2}$. In the same paper it is pointed out that $S_{3}$ has the structure of a distributive near-ring (see \#29, p. 342 of [9]). In this case $C=A_{3}$ and, in fact, $N / A_{3}$ is a unital ring. See also Theorem 2.6.

Setting $K(N)=$ set of distributive elements of $N$, Maxson showed ([6]) that $K(N)$ is a subnear-ring of $N$ precisely when P1 holds for all $a_{i} \in K(N), b_{i} \in N$, and in [3, Theorem 2] $K(N)$ was shown to be a normal subgroup. In fact we have 
Proposition 2.3. $K(N)$ is a subnear-ring and a left ideal when $N$ is pseudo-distributive.

Proof. If $x \in K(N)$, then for all $n_{i}, a_{i} \in N$

$$
\begin{array}{rlr}
{\left[n_{1}\left(n_{2}+x\right)-n_{1} n_{2}\right]\left(a_{1}+a_{2}\right)} & =n_{1}\left(n_{2}+x\right)\left(a_{1}+a_{2}\right)-n_{1} n_{2}\left(a_{1}+a_{2}\right) & \\
& =n_{1}\left[n_{2}\left(a_{1}+a_{2}\right)+x a_{1}+x a_{2}\right]-n_{1} n_{2}\left(a_{1}+a_{2}\right) \\
& =n_{1} n_{2}\left(a_{1}+a_{2}\right)+n_{1} x a_{1}+n_{1} x a_{2}-n_{1} n_{2}\left(a_{1}+a_{2}\right) & \text { by P2 } \\
& =n_{1} x a_{1}+n_{1} x a_{2} & \text { by P1 }
\end{array}
$$

On the other hand $\left[n_{1}\left(n_{2}+x\right)-n_{1} n_{2}\right] a_{1}+\left[n_{1}\left(n_{2}+x\right)-n_{1} n_{2}\right] a_{2}$ also equals $n_{1} x a_{1}+n_{1} x a_{2}$ by a straight forward calculation. Thus (I2) holds.

Note that $A \subseteq K(N)$.

In [3] it was shown that if $N$ is a simple pseudo-distributive near-ring then either $A=N$ and $N^{2}=0$, or $A=N$ is the finite field of order $p$, or $A=(0)$ and $N$ is a ring. In fact in the first case we also have $N=N^{\prime}$ (i.e. $(N,+)$ is a perfect group) since $A=N$ implies $K(N)=N$ so $D(N)=0$ and $C(N)=N^{\prime}=N$.

Recall [9, pp. 136-7] that in near-rings there are four "radicals" $J_{i}(N), i=0,1 / 2,1,2$ which generalize the Jacobson radical $J(R)$ of a ring. $J_{i}$ is an ideal for $i=0,1,2$.

Theorem 2.4. (a) $J_{i}(N / I)=J_{i}(N) / I$ for any ideal $I \subset J_{i}, i=0,1,2$.

(b) In a pseudo-distributive near-ring $N$ all the radicals coincide.

Proof. (a) Let $J$ represent any one of $J_{0}, J_{1}$ or $J_{2}$ and suppose $I$ is an ideal, $I \subseteq J$. Then the canonical surjection $f: N / I \rightarrow N / J$ yields, by $[9,5.13(\mathrm{~b})$ and 5.16], $f(J(N / I)) \subseteq J(f(N / I))=J(N / J)=0$. Therefore $J(N / I) \subset \operatorname{ker} f=J / I$. On the other hand, by $[9,5.15 \mathrm{c})], J(N / I) \supseteq J / I$ so equality holds as required.

(b) Since $J_{0} \subseteq J_{1} \subseteq J_{2}$, applying (a) we have $J_{i}\left(N / J_{0}\right)=J_{i}(N) / J_{0}$ for $i=0,1,2$. Since $C(N) \subseteq A \subseteq(L: N)$ for every left ideal $L$, therefore $C \subseteq J_{0}$ and hence $N / J_{0}$ is a ring. Thus all its radicals coincide and since $J_{0}\left(N / J_{0}\right)=0$ therefore $J_{i}(N)=J_{0}$ for $i=1,2$. Since $J_{\frac{1}{2}}$, (which, in general, is only a left ideal), lies between $J_{0}$ and $J_{1}$ therefore $J_{\frac{1}{2}}=J_{0}$ also.

We can then characterize $J(N)$ by a kind of "quasi-regularity". Recall that in a ring $R$, $x$ is right quasi-regular if $x+b+x b=0$ for some $b$, and $J(R)=\{x \mid x r$ is right quasiregular for all $r$.

Corollary 2.5. If $N$ is pseudo-distributive, $J(N)=\{x \mid$ for all $r$ there exists an such that for all $n, x r n+s n+x r s n=0\}$.

Proof. Applying Theorem 2.4(a) to $I=A \subset J$ and using Proposition 2.1(e) we see $J(N) / A$ is the set $\{x+A \mid(x+A)(r+A)$ is right quasi-regular for all $r \in N\}$. Therefore $J(N)=\{x \mid$ for all $r$ there exists $s$ such that $x r+s+x r s \in A\}$.

Now if $N$ is pseudo-distributive, $N / J$ is a ring with zero Jacobson radical. Suppose $N$ has DCCL (descending chain condition on left ideals). Then $([9,5.48])$ since all radicals 
coincide, $J$ is nilpotent. Since $N / J$ also has DCCL, it is a unital ring. Thus we have the following analogue to the Artin-Wedderbun Theorem for rings:

Theorem 2.6. If $N$ is a pseudo-distributive near-ring with $D C C L$, then $N / J$ is a unital ring which is a finite direct sum of matrix rings over skew fields.

\section{Matrix and group near-rings}

Let $N$ be pseudo-distributive and for any $S \subset N$ let $S_{k}$ denote the set of matrices in $N_{k}$ all of whose entries belong to $S$. Let $\mathscr{J}(N)$ be the set of ideals of $N$.

Proposition 3.1. There is a mapping $T: \mathscr{H}(N) \rightarrow \mathscr{J}\left(N_{k}\right)$ and a mapping $S: \mathscr{J}\left(N_{k}\right) \rightarrow \mathscr{J}(N)$ such that $S T=\mathrm{id}$ (so $T$ is $1-1$ and $S$ is onto).

Proof. Clearly if $J$ is an ideal in $N, J_{k}$ is an ideal in $N_{k}$ so $T(J)=J_{k}$. Conversely, if $\mathscr{J}$ is an ideal in $N_{k}$, set $S(\mathscr{J})=\left\{n \mid n=a_{11}\right.$ for some $\left.\left(a_{i j}\right)=A \in \mathscr{J}\right\}$. Clearly $S(\mathscr{J})$ is a normal subgroup of $N$. Moreover if $n \in S(\mathscr{J})$ let $X=\left(\delta_{i j} x\right)$ and $Y=\left(y_{i j}\right)$ where $y_{11}=y, y_{i j}=0$ for $(i, j) \neq(1,1)$. Then $x(y+n)-x y$ is the $1-1$ entry of $X(Y+A)-X Y \in \mathscr{J}$. Since $S(\mathscr{J})$ is clearly right $N$-closed, it is an ideal.

Next we have $J \subseteq S T(J)$ trivially and if $n$ is the 1-1 entry of some matrix in $J_{k}$, clearly $n \in J$.

We note that in the case of unital rings $T S=$ id also, since by using matrix units one can show that if $A=\left(a_{i j}\right) \in \mathscr{J}$ then $a_{i j}$ is the 1-1 entry of some matrix in $\mathscr{J}$. In the present case, let $X_{i j}$ be the matrix with entry $x$ in position $i-j$ and zeros elsewhere. If $A \in \mathscr{J}$, then $B=\sum_{k} X_{k i} A Y_{j k} \in \mathscr{J}$ and $B$ is the matrix with $x a_{i j} y$ all along the diagonal. Thus for all $i, j$, and all $x, y$, we have $x a_{i j} y \in S(\mathscr{F})$. This also shows that every ideal in $N_{k}$ intersects $N$ non-trivially (where $N$ embeds canonically in $N_{k}$ ).

Lemma 3.2. If $I$ is an ideal in $N,(N / I)_{k}$ is isomorphic to $N_{k} / I_{k}$.

Proof. The map $N_{k} \rightarrow(N / I)_{k}$ sending $\left(a_{i j}\right)$ to $\left(a_{i j}+I\right)$ is a surjection with kernel $I_{k}$.

As a result $D_{k} \supseteq D\left(N_{k}\right)$ and $C_{k} \supseteq C\left(N_{k}\right)$. We note in passing that $A_{k}=A\left(N_{k}\right)$ and $K\left(N_{k}\right)=$ $K(N)_{k}$.

Let $J(N)$ be the Jacobson radical. For rings it is well known that $J\left(N_{k}\right)=J(N)_{k}$. Using this we can show

Theorem 3.3. If $N$ is pseudo-distributive, $J\left(N_{k}\right)=J(N)_{k}$.

Proof. By the lemma, $N_{k} / J_{k} \simeq(N / J)_{k}$ and since $N / J$ is a ring $J\left((N / J)_{k}\right)=(J(N / J))_{k}=0$. Then $0=J\left(N_{k} / J_{k}\right) \supseteq J\left(N_{k}\right)+J_{k} / J_{k}$ by $\left.[9,5.15 \mathrm{c})\right]$ so $J\left(N_{k}\right) \subseteq J_{k}$. Now consider the ring $N / C$. Using Theorem 2.4 and the lemma we have $J\left(N_{k}\right) / C_{k} \simeq J\left(N_{k} / C_{k}\right) \simeq J\left((N / C)_{k}\right) \simeq$ $(J(N / C))_{k} \simeq(J(N) / C)_{k} \simeq J_{k} / C_{k}$. Combined with the fact $J\left(N_{k}\right) \subseteq J_{k}$, we have equality.

Turning now to group near-rings, we recall the comments made in the introduction that $N G$ is taken to be the set of functions $\phi: G \rightarrow N$ with finite support, and multiplication defined by $\left(\phi^{*} \alpha\right) g=\sum_{x} \phi(x) \alpha\left(x^{-1} g\right)$. $N G$ is then an $N$-group, and $N$ is a subnear-ring of $N G$ via the functions $\hat{n}$ where $\hat{n}(e)=\hat{n}$ and $\hat{n}(g)=0$ for $g \neq e$ (we note in 
passing that this embedding depends on the zero-symmetry of $N$ ). $N$ is also a normal subgroup of $(N G,+)$.

The standard theory of unital group rings $R G$ (see eg. [1] or [8]) makes extensive use of the fact that $G$ can also be embedded in $R G$ using the identity of $R$. In particular, this allows one to say that $\phi \cdot g$ and $g \cdot \phi \in R G$ for all $\phi \in R G, g \in G$. In the pseudo-distributive near-ring case we can define left and right $G$-actions on $N G$ as follows: $(\phi \circ g)(h)=\phi\left(h g^{-1}\right)$ and $(g \circ \phi)(h)=\phi\left(g^{-1} h\right)$. Then for all $\alpha, \beta \in N G$ and $g, g_{1} \in G$ we have

$$
\begin{array}{rlrl}
\left(\alpha^{*} \beta\right) \circ g & =\alpha^{*}(\beta \circ g) & g \circ\left(\alpha^{*} \beta\right) & =(g \circ \alpha)^{*} \beta \\
(\alpha+\beta) \circ g & =\alpha \circ g+\beta \circ g & g \circ(\alpha+\beta) & =g \circ \alpha+g \circ \beta \\
\alpha \circ g g_{1} & =(\alpha \circ g) \circ g_{1} & g g_{1} \circ \alpha & =g \circ\left(g_{1} \circ \alpha\right) \\
(\alpha \circ g)^{*} \beta & =\alpha^{*}(g \circ \beta) & g \circ\left(\alpha \circ g_{1}\right) & =(g \circ \alpha) \circ g_{1}
\end{array}
$$

Also $\hat{n} \circ g=g \circ \hat{n}$ for all $n \in N, g \in G$.

It follows that each $\alpha \in N G$ can be written as $\alpha=\sum_{g} \hat{n}_{g} \circ g$ where $n_{g}=\alpha(g)$. In what follows we shall generally indicate both* and $\circ$ by simple juxtaposition.

A left ideal $I$ of $N G$ will be called left (right) $G$-closed if $g I \subseteq I(I g \subseteq I)$ for all $g \in G$. For example Ann $N G$ is left and right $G$-closed (see Theorem 3.4) and since $J(N G)=$ $\cap(L: N G)$ where $L$ is an $i$-modular left ideal $[9$, p. 136] it follows that $J(N G)$ is right $G$ closed. Note that $I$ is left and right $G$-closed if $\alpha \in I$ implies $\beta \in I$ for all $\beta$ with range $\alpha=$ range $\beta$. Writing Supp $\phi=\{g \mid \phi(g) \neq 0\}$ for the support of $\phi$ we have

1. $\operatorname{Supp}(g \phi)=g \operatorname{Supp} \phi$

2. If Supp $\alpha \cap \operatorname{Supp} \beta=\emptyset$ then

$$
\phi(\alpha+\beta)=\phi \alpha+\phi \beta \text { for all } \phi \in N G
$$

3. If $x \in \operatorname{Supp}(\alpha \beta)$, there exist $h \in \operatorname{Supp} \alpha$ and $g \in \operatorname{Supp} \beta$ such that $x=h g$.

Theorem 3.4. (a) If $I$ is a (left) ideal of $N$ then $I G=\{\phi \in N G \mid \phi(x) \in I$ for all $x \in G\}$ is a G-closed (left) ideal of $N G$. (b) If $I$ is two-sided $N G / I G \simeq(N / I) G$ as near-rings. (c) $K(N G)=K(N) G, \operatorname{Ann}(N G)=(\operatorname{Ann} N) G, C(N G)=C(N) G$ and $D(N G)=D(N) G$. (d) The map $T: \mathscr{J}(N) \rightarrow \mathscr{J}(N G)$ given by $T(I)=I G$ and the map $S: \mathscr{J}(N G) \rightarrow \mathscr{J}(N)$ given by $S(\mathscr{J})=$ $\mathscr{J} \cap N$ satisfy $S T(I)=I$ (cf. Proposition 3.1).

Proof. (a) Clearly $(I G,+)$ is a normal subgroup of $N G$. Moreover

$$
\begin{gathered}
{[\alpha(\beta+\phi)-\alpha \beta](\mathrm{g})=\sum_{x} \alpha(x)(\beta+\phi)\left(x^{-1} g\right)-\sum_{x} \alpha(x) \beta\left(x^{-1} g\right)=\sum_{x} \alpha(x)\left(\beta\left(x^{-1} g\right)+\phi\left(x^{-1} g\right)\right)} \\
-\alpha(x) \beta\left(x^{-1} g\right) \quad(\text { by P1) }
\end{gathered}
$$

which is in $I$ for all $\alpha, \beta \in N G, \phi \in I G$. Also $\phi \in I G$ implies $\alpha \in I G$ for all $\alpha$ with range $\alpha=$ range $\phi$ so $I G$ is left and right $G$-closed. 
(b) If $I$ is a two sided ideal and $\pi: N \rightarrow N / I$ the canonical map, define $f_{1}: N G \rightarrow N / I G$ by $f_{1}(\phi)=\pi \circ \phi$. Then $f_{1}$ is a near-ring surjection with kernel $I G$.

(c) If $\phi \in K(N G)$ then $\phi(\alpha+\beta)=\phi \alpha+\phi \beta$ for all $\alpha, \beta$. Therefore $\sum_{x} \phi(x)(\alpha+\beta)\left(x^{-1} g\right)=$ $\sum_{x} \phi(x) \alpha\left(x^{-1} g\right)+\phi(x) \beta\left(x^{-1} g\right)$ for all $g \in G$. In particular $\alpha$ and $\beta$ can be chosen to have singleton support $x^{-1} g$ and arbitrary values $n, n^{\prime} \in N$ so that for all $x \in G$, all $n, n^{\prime} \in N, \phi(x)\left(n+n^{\prime}\right)=\phi(x) n+\phi(x) n^{\prime} \quad$ whence $\quad \phi \in K(N) G$. The reverse inclusion $K(N) G \subset K(N G)$ is clear, and the proofs for $D(N G), C(N G)$ and $A(N G)$ are straightforward.

(d) Is easily shown.

We next investigate the connection between subgroups of $G$ and $G$-closed left ideals in $N G$. If $H$ is any subgroup of $G$, the set of cosets $G / H$ can be used to define an $N$-group $N G / H$ which will be a near-ring if $H$ is normal. We first consider the case when $N$ is a ring so $N G / H$ is an $N$-module.

Theorem 3.5. Let $\mathrm{N}$ be a ring. The mapping $f_{2}: N G \rightarrow N G / H$ given by $\left(f_{2} \phi\right)(g H)=$ $\sum_{h \in H} \phi(g h)$ is an $N$-module homomorphism whose kernel $\omega H$ is a left ideal which is left $G$-closed. Moreover $\omega H$ is additively generated by $S=\{\phi \circ h-\phi \mid \phi \in N G, h \in H\}$.

Proof. The first part is a normal ring theoretic proof. $\omega H$ is left $G$-closed because $\sum_{h}(x \circ \phi)(g h)=\sum_{h} \phi\left(x^{-1} g h\right)=0$. Note that if $H$ is normal, $\omega H$ is also right $G$-closed. Clearly $S \subset \operatorname{ker} f_{2}$ since

$$
\sum_{h_{i} \in H}(\phi \circ h-\phi)\left(g h_{i}\right)=\sum_{h_{i} \in H} \phi\left(g h_{i} h^{-1}\right)-\phi\left(g h_{i}\right)=0 .
$$

Conversely consider the case $H=G$. Then if $\phi \in \omega G, \sum_{g} \phi(g)=0$ and without loss of generality $e \in \operatorname{Supp} \phi$ so define

$$
\begin{aligned}
\alpha_{i}(e) & =\phi\left(g_{i}\right) \quad \text { for } \quad i=1, \ldots, k \\
& =0 \text { otherwise }
\end{aligned}
$$

Then

$$
\begin{gathered}
\left(\alpha_{i} \circ g_{i}-\alpha_{i}\right)(e)=-\phi\left(g_{i}\right) \\
\left(\alpha_{i} \circ g_{i}-\alpha_{i}\right)\left(g_{i}\right)=\phi\left(g_{i}\right)
\end{gathered}
$$

and

$$
\left(\alpha_{i} \circ g_{i}-\alpha_{i}\right)\left(g_{j}\right)=0 \text { for } g_{j} \neq g_{i}, e
$$


Thus

$$
\begin{aligned}
\sum_{i}\left(\alpha_{i} \circ g_{i}-\alpha_{i}\right)(x) & =\phi\left(g_{i}\right) \quad \text { if } x=g_{i} \\
& =-\sum_{i} \phi\left(g_{i}\right)=\phi(e) \text { if } x=e
\end{aligned}
$$

so $\phi=\sum_{i}\left(\alpha_{i} \circ g_{i}-\alpha_{i}\right)$ as required.

The proof for a proper subgroup $H$ follows similarly.

The proof also shows that $\omega G$ is additively generated by $T=\{g \circ \phi-\phi \mid g \in G, \phi \in N G\}$ and therefore $\omega G$ is a right $G$-closed right ideal, i.e. an ideal. The same holds for $\omega H$ where $H$ is any normal subgroup of $G$. Continuing with the assumption that $N$ is a ring, to each left $G$-closed left ideal $J$ of $N G$ we can associate a subset $\Omega J=\{g \mid \phi \circ g-\phi \in J\}$ of $G$. In fact $\Omega J$ is a subgroup since $\phi \circ g g_{1}-\phi=\phi \circ g \circ g_{1}-\phi \circ g+\phi \circ g-\phi$ and $\phi \circ g^{-1}-$ $\phi=-(\alpha \circ g-\alpha)$ where $\alpha$ is defined by $\alpha=\phi \circ g^{-1}$. Thus

Proposition 3.6. When $N$ is a ring, there is a mapping $\omega:\{$ Subgroups of $G\} \rightarrow\{G$-closed left ideals of $N G\}$ and a mapping $\Omega$ in the reverse direction such that $\Omega \omega H=H$. Thus $\omega$ is $1-1$ and $\Omega$ is onto.

Now we consider $N G$ for any pseudo-distributive near-ring $N$. If $H$ is normal in $G$ with canonical map $\sigma: G \rightarrow G / H$, then there is a natural map $\bar{\sigma}(\phi): N G / H \rightarrow N G$ given by $\bar{\sigma}(\phi)=\phi \circ \sigma$. In fact $\bar{\sigma}$ is a near-ring monomorphism by which we can identify $N G / H$ with a subnear-ring of $N G$, namely $\{\phi \in N G \mid \phi(g h)=\phi(g)$ for all $h \in H\}$. Also there are natural maps $\theta_{H}: N G \rightarrow N H$ (given by restriction) and $\rho_{H}: N H \rightarrow N G$ (where $\rho_{H}(\phi)=\phi$ on $H$ and $\rho_{H}(\phi)=0$ on $\left.G-H\right)$ such that $\theta_{H \rho H}=$ id. In fact, $\rho_{H}$ is a normal map so $N H$ is a direct summand of $N G$ (as $N$-groups).

If $I$ is any ring ideal in $N$, and $H$ is normal we have from Theorems 3.4 and 3.5 the composite maps $f_{2} f_{1}: N G \rightarrow N / I G \rightarrow N / I G / H$ whose kernels $\omega_{I} H$ are ideals in $N G$. Taking $H=G$ we get the

Corollary. $N G$ contains ideals $\omega_{I} G$ for which $N G / \omega_{I} G \simeq N / I$ as rings.

Note that $\omega_{I} G=\left\{\phi \in N G \mid \sum_{g \in G} \phi(g) \in I\right\}$.

This is a key result, corresponding to the fact for unital group rings that the augmentation ideal $\Delta$ of $R G$ satisfies $R G / \Delta \simeq R$. (See also [7], Theorem 4.9). This leads to several results transferring properties from $R$ to $R G$ with appropriate conditions on G. If $N$ is pseudo-distributive and abelian (eg. examples (1) and (2) in [4]) then $\delta: N G \rightarrow N$ given by $\delta(\alpha)=\sum_{g} \alpha(\mathrm{g})$ is still well defined and gives $N G / \operatorname{ker} \delta \simeq N$. In the more general case the above result allows some transfer of properties between $N G$ and $N / I$ for ring ideals $I$ as we shall see below. Moreover, Theorem 3.4 shows how certain factors of $N G$ are group rings $(N / I) G$, and indeed they may even be unital as the example following Proposition 2.2 shows. We repeat that certain conditions imposed on the pseudo-distributive near-ring $N G$ (eg. Von Neumann regularity, the absence of nonzero nilpotent ideals) would force $N G$ to be a ring; on the other hand some information about non-unital group rings can be obtained in this way. For example, using a proof like that for unital rings one can show that if $N G$ is regular, then $N$ is regular and $G$ is locally finite. We give a sample result for general pseudo-distributive group rings: 
Proposition 3.7. If $N G$ has $D C C L$, then $N / I$ is a left artinian ring for all ring ideals $I$, and $G$ is finite. Moreover, $N / J(N)$ is a unital artinian semi-simple ring. If $N$ is abelian, $N$ is also left artinian.

Proof. $N G / \omega_{I} G \simeq N / I$ is left artinian for all ring ideals $I . N G / J G \simeq N / J G$ is an artinian ring and, as noted earlier, $N / J$ has no nilpotent ideals. Since $N / J \simeq(N / J) G / \Delta$ is also left artinian, therefore $N / J$ has an identity. Then the fact that $N / J G$ is artinian implies $G$ is finite [1, Theorem 1$]$.

Now suppose $G$ is finite. Let $S$ be the set of constant maps $\bar{n}: G \rightarrow N$ where $\bar{n}(g)=n$ for all $g \in G$. In unital group rings, $S$ is an ideal equal to $\mathrm{Ann}^{l} \omega G$ and $\mathrm{Ann}^{r} \omega G$ ([1]).

Proposition 3.8. (a) If $N$ has a left cancellable element than $S=\mathrm{Ann}^{r} \omega_{I} G$ for all ring ideals $I \subseteq A$. (b) If $N$ is distributive $\mathrm{Ann}^{l} \omega_{c} G \supseteq S$. (c) If $N$ is a ring, $S$ is an ideal contained in both $\mathrm{Ann}^{l} \omega_{c} G$ and $\mathrm{Ann}^{r} \omega_{c} G$, which are also ideals.

Proof. (a) Note that if $N$ has a right cancellable element, it is a ring [3]. Certainly $S \subseteq \mathrm{Ann}^{r} \omega_{I} G$ since $\phi \cdot \bar{n}=\sum_{x} \phi(x) n=\left[\sum_{x} \phi(x)\right] n=0$ when $\sum_{x} \phi(x) \in I \subseteq A$. Conversely, if $\phi \alpha=0$ for all $\phi \in \omega_{I} G$ then $\sum \phi(x) \alpha\left(x^{-1} g\right)=0$ for all $g$ so in particular $\sum \phi(x) \alpha\left(x^{-1}\right)=0$. If $\alpha$ is not constant there exist $y_{1}, y_{2}$ such that $\alpha\left(y_{1}\right) \neq \alpha\left(y_{2}\right)$. Define $\phi$ by $\phi\left(y_{1}^{-1}\right)=n, \phi\left(y_{2}^{-1}\right)=$ $-n, \phi(g)=0$ for all other $g$. Then $\phi \in \omega_{I} G$ since in fact $\sum_{x} \phi(x)=0$ and $n \alpha\left(y_{1}\right)-n \alpha\left(y_{2}\right)=0$. Thus if $n$ is left cancellable, $\alpha\left(y_{1}\right)=\alpha\left(y_{2}\right)$ which is contradiction.

(b) Since $\omega_{c} G=\left\{\sum_{g} \phi g-\phi+C G\right\}$ and since $N$ distributive implies $C=N^{\prime}$ therefore if $\left(\bar{n} \in S, \bar{n} \cdot(\phi g-\phi+\alpha)(y)=\sum_{x \in G} n[\phi \cdot g(x)-\phi(x)+\alpha(x)]=\sum_{x} n \phi\left(x g^{-1}\right)-n \phi(x)+\sum_{x} n \alpha(x)\right.$ for all $\alpha \in C G$. The first sum is zero since as $x$ runs through Supp $\phi$ so does $x g^{-1}$ and the second sum is zero since $N \cdot N^{\prime}=0$ in the distributive case.

(c) This is straight forward.

We conclude with the following observations: When $(N, S)$ is a distributively generated near-ring the $N$-groups of interest are the $(N, S)$-groups, i.e. those $N$-groups on which $S$ acts distributively (see eg. [9, p. 182] and [7]). By analogy, when $N$ is pseudo-distributive define an $N$-group $A$ to be a p.d. $N$-group if

$$
\begin{aligned}
& \text { (PD1) } n_{1} a_{1}+n_{2} a_{2}=n_{2} a_{2}+n_{1} a_{1} \\
& \text { (PD2) } n_{1}\left(n_{2} a_{2}+n_{3} a_{3}\right)=n_{1} n_{2} a_{2}+n_{1} n_{3} a_{3} \text { for }
\end{aligned}
$$

all $n_{i} \in N, a_{i} \in A$. That is, $N A$ is an abelian $N$-subgroup of $A$ on which $N$ acts distributively. Examples are $N$ itself, any left ideal of $N, N / I$ for $I$ any left ideal containing $C(N), N_{k}$, and $N G$.

Now classical group algebras were used to study the representation of a group as a group of matrices. For $(N, S)$-groups, Meldrum [7] was able to establish a 1-1 correspondence between representations of $G$ as a group of $(N, S)$-automorphisms and representations of the d.g. group near-ring. In the pseudo-distributive case there is clearly no hope of restricting representations of $N G$ to $G$ since $G$ is not identified with a subgroup of $N G$. However, if $\mu: G \rightarrow N_{k}$ is a semi-group homomorphism to the multiplicative structure of $N_{k}$, then $\mu$ induces a "representation" $\hat{\mu}: N G \rightarrow N_{k}$ defined by $\hat{\mu} \phi=\sum_{g} \phi(g) \mu(g)$. Since $N_{k}$ is a p.d. $N$-group, $\hat{\mu}$ is a near-ring homomorphism. 
Acknowledgement. The author gratefully acknowledges the support of the NSERC of Canada.

\section{REFERENCES}

1. I. G. Connell, On the group ring, Canad. J. Math. 15 (1963), 650-685.

2. H. Heatherly, Distributive near-rings, Quart, J. Math. Ser. (2) 24 (1973), 63-70.

3. H. Heatherly and S. Ligh, Pseudo-distributive near-rings, Bull. Aust. Math. Soc. 12 (1975), $449-456$.

4. S. Ligh, A note on matrix near-rings, J. London Math. Soc. (2) 11 (1975), 383-384.

5. G. MAson, Solvable and nilpotent near-ring modules, Proc. Amer. Math. Soc. 40 (1973), 351-357.

6. C. Maxson, On near-rings and near-ring modules (Doctoral dissertation, SUNY at Buffalo, 1967).

7. J. Meldrum, The group distributively generated near-ring, Proc. London Math. Soc. (3) 32 (1976), 323-346.

8. D. Passman, Infinite group rings (Marcel Dekker, New York, 1971).

9. G. Pilz, Near-rings (North Holland, Amsterdam, 1977).

Department of Mathematics \& Statistics

UNIVERSITY OF NEW BRUNSWICK

Fredericton, N. B., CANADA 